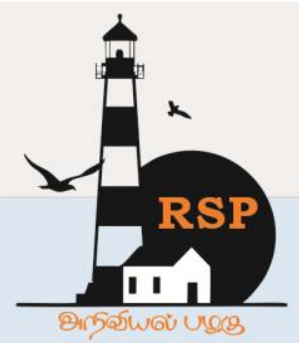

INTERNATIONAL RESEARCH JOURNAL ON ADVANCED SCIENCE HUB

RSP SCIENCE HUB

(The Hub of Research Ideas)

Available online at www.rspsciencehub.com

\title{
Pick and Place Robot for Surface Mounting Devices
}

Ramanathan $S^{1}$, Akash $V^{2}$, Arun Prasad $R^{3}$, Venkat Rahul $M^{4}$,

${ }^{1}$ Assistant Professor, Dept. of Mechanical Engineering, Sri Sairam EngineeringCollege, Tamilnadu, India

${ }^{2}$ Student, Dept. of Mechanical Engineering, Sri Sairam EngineeringCollege, Tamilnadu, India

${ }^{3}$ Student, Dept. of Mechanical Engineering, Sri Sairam EngineeringCollege, Tamilnadu, India

${ }^{4}$ Student, Dept. of Mechanical Engineering, Sri Sairam Engineering College, Tamilnadu, India venkatrahul1998@gmail.com ${ }^{4}$

\begin{abstract}
This project is based on pick-and-place machines for placing surface mount devices onto the printed circuit board. In general, the SMT pick and place robot using an end effectors to pick and place the component on the PCB and a controller is used to control the movement of the stepper motor. Our SMT pick and place machine is designed with a Cartesian configuration, and the Arduino UNO controller with the CNC shield controls the stepper motors. The end effectors use a vacuum suction pen to pick and place the components and a belt mechanism is used in the z-axis for up-down motion. Our machine is portable and well suited for small scale industries. Our project satisfies one important sustainable development goal - industry, innovation, and infrastructure.
\end{abstract}

Keywords: SMT, PCB, Arduino UNO, Stepper Motor, etc

\section{Introduction}

Generally, a pick-and-place machine is used to pick and transfer a specific component to any desired location. The pick and place machine's greatest significance are its precision, accuracy, reduced time, and less manpower. This is where we are using this machine to place Surface Mount Devices on the Printed Circuit Board (PCB). Surface Mount Technology's evolution in soldering over pinhole soldering demands the use of automation for the process in question.

\subsection{Surface Mount Technology}

Surface Mount Technology is an area of electronic assembly used to mount Electronic components to the surface of the printed circuit board (PCB) instead of inserting elements through holes like typical assembly. SMT was developed to scale back producing prices and additionally to create additional economical use of the PCB area. As a result of the introduction of surface mount technology, it's currently attainable to create extremely advanced electronic circuits into smaller and smaller assemblies with smart repeatability thanks to the upper level of automation [1-4].

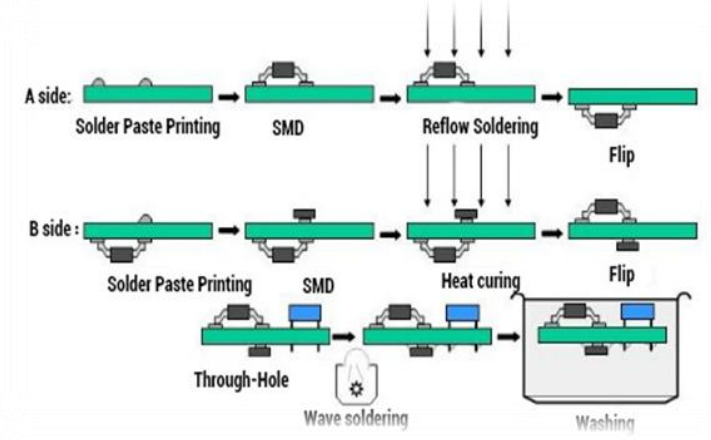

\subsection{Pick and Place Machine}

Pick and place of Surface Mount Device machine 
is highly essential to implement. This machine assists in placing such small components in the Circuit Boards. Major types of pick and place machines (PNP) are

- Multi-head PNP

- Turret type PNP

- Sequential PNP

Our machine is a sequential PNP which involves a feeder mechanism for transferring components that acts similar to a conveyor.

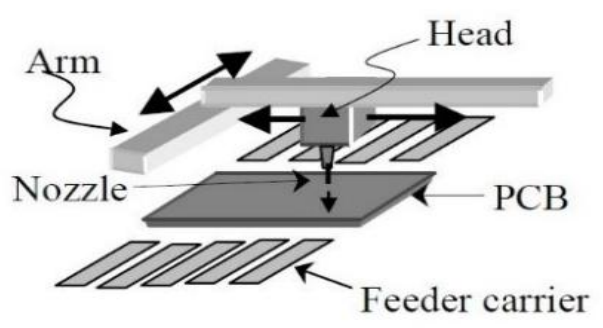

A sequential pick-and-place SMD placement machine.

\subsection{Purpose of our Machine}

The purpose of our machine is to ensure the placement of SMD components with high accuracy and to make the machine so compact and portable. [5-7]

\section{Related Works}

\subsection{Literature Study}

A literature study is made on similar pick and place machines which differs in the application and comparison is made on different papers. From the observations, we summarised the existing work, and the required modifications are made accordingly.

1) International Journal Of Science, Engineering And Technology Research (Ijsetr) Paper On Pick And Place Robotic Arm Using Arduino By Harish K, Megha D, Shuklambari M, Amit K, Chaitanya K Jambotkar

For the application of packaging pick and place robot using Arduino mega. This is helpful for the Palletising application.
2) International Journals Of Environment And Sustainable Development (Ijesd) Paper On Multihandling Pick And Place Robot By S Premkumar, K Surya Varman

The machine involves a combination of gripper and vacuum suck mechanism and Motion controlled using revolute joints for lifting, gripping, and placing several parts. This is a Multihead type PNP.

3) International Journal of Engineering Research And Development (Ijerd) Paper On Design Analysis Of A Remote Controlled "Pick And Place" Robotic Vehicle By B.O. Omijeh, R. Uhunmwangho, M. Ehikhamenle Department Of

Electrical Engineering, University Of Port Harcourt

The arm containing 5 degrees of freedom and wheels for the motion of the vehicle, this robot is remotely controlled for handling hazardous objects. Such robots usually have a SCARA configuration.

\subsection{Summary on the Literature Study}

Most of the above research was only conducted on SCARA, even though they are flexible than a Cartesian robot, SCARA can only lift limited payload which itself includes its motors. On the contrary Cartesian robots can handle larger payload and provide better accessibility to a variety of parts.Regarding the end effector, most of the existing machines use gear mechanism for the $\mathrm{z}$ translation movement that has been changed into a pulley belt mechanism to eliminate the wear

\section{Proposed Work}

\section{Design}

The finalized design involves the integration of various mechanisms assembled altogether and also the bottom-up assembly approach is enforced.

\section{Bottom-Up Assembly}

Users start designing a product in the Bottom-up approach by detailing its low-level component. 
www.rspsciencehub.com

Then, these are grouped into distinct components. The process is carried on until the product itself is formed.

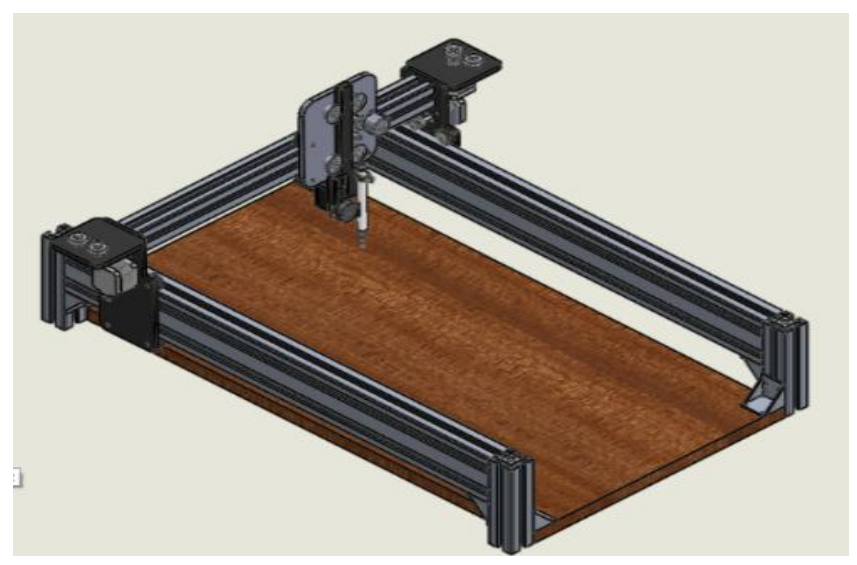

Fig.1.The 3D isometric view of our project

\subsection{Major Mechanisms}

\subsubsection{Vacuum Suction}

The core of our project is designing and developing a pick and place machine that is accomplished through a vacuum suction pump. The pressure required to suck the part (PICK) is given and the part (PLACE) is dropped.

\subsubsection{Feeder Mechanism}

To move the SMD elements from a strip after a certain delay time, the feeder mechanism is used. The required delay time is set for a particular sequence.

\subsubsection{Belt Mechanism}

V-belt and pulley mechanisms are employed altogether for three axes of motion. The primary motion is attained by the stepper motors which is thereby transmitted to the $\mathrm{X}, \mathrm{Y}, \mathrm{Z}$ frames (motion axes) with the assistance of a pulley and a belt.

\subsection{Components Used in Our Machine}

\subsubsection{Aluminium Extrude}

The profile of the Cartesian is designed with Aluminium extrusion. Profile used - 4040, 4080.
Volume 02 Issue 08 August 2020

Aluminium T - Profile Grade 6005 is an alloy in the wrought aluminium-magnesium-silicon family. Its chemical composition includes, Aluminium: 97.5 to $99.0 \%$, Chromium: $0.1 \%$, Copper: $0.1 \%$, Iron: $0.35 \%$, Magnesium: 0.4 to $0.6 \%$, Manganese: $0.1 \%$, Silicon: 0.6 to $0.9 \%$, Titanium: $0.1 \%$, Zinc: $0.1 \%$, Residuals: $0.15 \%$. The dimensions are 20x40mm.

\subsubsection{Stepper Motor}

The NEMA 17 motor with a step angle of 1.8 degrees and a torque of $3.2 \mathrm{Kg}$ is used for all 3 axis movements. A stepper motor is an electric motor that divides the rotation in terms of steps, energizing each phase in series, one stage at a time. Without any position sensor, the motor can be moved to one of those steps. Motor selection is based on torque, step degree and power rating.

\subsubsection{Arduino Controller}

Arduino UNO controller is used for controlling the stepper motor in all the axes. It can operate at a voltage of 5V. It has 14 Digital Input /Output pins (of which 6 provide PWM output). It requires 20 $\mathrm{mA}$ DC Current per I/O pin and $50 \mathrm{~mA}$ DC for $3.3 \mathrm{~V}$ pin. The available flash memory is $32 \mathrm{~KB}$ of which $0.5 \mathrm{~KB}$ used by the boot loader. It has a clock speed of sixteen megacycles per second.

\subsubsection{CNC Shield}

Arduino UNO is used for stepper motor control in all axes. It can operate at 5V. It has 14 Digital Input / Output pins (6 of which give output from $\mathrm{PWM}$ ). For $3.3 \mathrm{~V}$ pin, it requires $20 \mathrm{~mA} \mathrm{DC}$ Current per I / O pin, and $50 \mathrm{~mA}$ DC. The available flash memory is $32 \mathrm{~KB}$ and the boot loader uses $0.5 \mathrm{~KB}$ of that. It has an average clock speed of 16 megacycles per second.

\subsubsection{Vacuum Pump}

This is the most important component required to create the suction pressure required to pick and place the component.

Rated Voltage: DC 6V - 12V 
Working Current :> 1.5A

Flow Rate: 1.5 LPM

Maximum Pressure: 5.5 Bar

Noise Level: $55 \mathrm{~dB}$

Pump Type: Piston Pump

Nozzle Diameter: $4.8 \mathrm{~mm}$

\subsection{Software Used}

\subsubsection{Arduino}

The Arduino Integrated Development Environment (IDE) is an application written in java programming language. It has a code editor with features such as text search and replacement, automatic indenting, brace matching, and highlighting syntax, related to it provides easy one-click mechanisms for compiling and transferring programs to an Arduino board. It includes message area, a text screen, and a toolbar with common function buttons.

\subsubsection{UGS (Universal G-Code Sender)}

Universal G-code Sender prevails as a selfcontained Java application with all external dependencies, meaning if you have the Java Runtime atmosphere setup that UGS provides for the rest. The Universal G-code Sender turns out to be an open-source G-code sender compatible with Java-based GRBL. This program is used to run a $\mathrm{CNC}$ machine that is controlled by GRBL.

\subsubsection{Features}

- It's been tested on Windows, Linux and Raspberry Pi.

- The JAR file contains Native Dependencies for all OS supported.

- Estimates on duration.

- More than 3000 lines of the unit look at the code, and another 1,000 lines of comments documenting the tests.

- Optimization of G-Code configurable.

\subsection{Calculation}

\subsubsection{Motor Torque Calculation}

- Weight of Al Extrusion - 1.036kg.
- Acrylic used $-0.548 \mathrm{~kg}$.

- 3D printed part- $0.66 \mathrm{~kg}$.

- 17HS2408Stepper Motor- 0.150kg. Vacuum pen- $0.034 \mathrm{~kg}$.

- Total weight $- \pm 2.5 \mathrm{~kg}$ (500g tolerance).

- We need stepper to move $3 \mathrm{kgs}$ approximately in the $\mathrm{x}$-axis and $1 \mathrm{~kg}$ in the $\mathrm{y}$-axis.

- The required holding torque of the motor should be 3 to $4 \mathrm{~kg}-\mathrm{cm}$.

- Two 17HS3401 stepper motor with holding torque of $2.6 \mathrm{~kg}-\mathrm{cm}$ in the $\mathrm{X}$-axis is chosen.

- One 17HS3401 is chosen for the y-direction. For Z-axis 17HS2408 stepper with a torque of $1.22 \mathrm{~kg}-\mathrm{cm}$ is used.

\subsubsection{Rated Current Calculation (To be set in Arduino)}

To set this limit is to calculate the reference voltage that corresponds to your required current limit then alter this limit potentiometer till you live that voltage on the VREF pin. The IMAX, current limit, relates to the reference voltage as follows

IMAX=VREF / ( $8 \times$ RCS $)$ or,

Rearranged to solve for VREF:

$\mathrm{VREF}=8 \times \mathrm{IMAX} \times \mathrm{RCS}$

RCS is the current sense of resistance. Our stepper motor rated current is $1.3 \mathrm{~A} \& 0.6 \mathrm{~A}$

We use the A4988 stepper driver, for this purpose we have to set Vref to the desired level to get the rated current.

Current limit $=$ VREF x 2.5

$\mathrm{VREF}=1.3 / 2.5=0.52$

$\mathrm{VREF}=0.6 / 2.5=0.24$

\subsubsection{V-Belt Calculation}

Grade GT2 open loop timing belt. The material used is Neoprene Rubber with Fiberglas Core. The pitch of the belt is $2 \mathrm{~mm}$. It has a width of $6 \mathrm{~mm}$ and a total length of $2 \mathrm{~m}$.

\subsubsection{Vacuum Suction Pressure}

The work piece is lifted vertically and horizontally transported. Acceleration is about 5 $\mathrm{m} / \mathrm{s}^{2}$. And $\mathrm{m}=140 \mathrm{~g}$. 

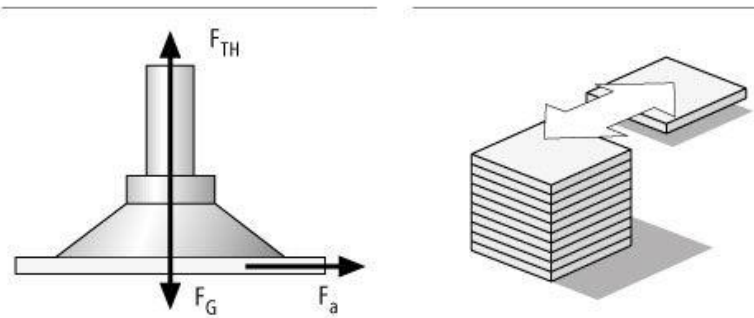

Fig.2.The suction-head land on a work piece horizontally that is to be moved aside.

$\mathbf{F}_{(\mathbf{T H})}=\mathbf{m} \times(\mathbf{g}+\mathbf{a} / \boldsymbol{\mu}) \times \mathbf{S}$.

$\mathrm{F}_{(\mathrm{TH})}=$ theoretical holding force $[\mathrm{N}]$.

$F_{(a)}=$ Acceleration force $=\mathrm{m} \times$ a.

$\mathrm{m} \quad=$ Weight $[\mathrm{kg}]$.

$\mathrm{g} \quad=$ Gravity $\left[9.81 \mathrm{~m} / \mathrm{s}^{2}\right]$.

a $\quad=$ Acceleration $\left[\mathrm{m} / \mathrm{s}^{2}\right]$ of the system

(keep in mind Emergency Stop situations).

$\mu \quad=$ Friction coefficient.

$\mathrm{S}=$ Safety.

\subsubsection{Our Calculation}

$\mathrm{F}_{(\mathrm{TH})}=0.140 \times\left(9.81 \mathrm{~m} / \mathrm{s}^{2}+5 \mathrm{~m} / \mathrm{s}^{2} / 0.6\right) \times 2$.

$\mathrm{F}_{(\mathrm{TH})}=4.85 \mathrm{~N}$.

Pressure $=$ Force $/$ Area.

$\mathrm{P}=4.85 / 25=0.194 \mathrm{~N} / \mathrm{mm}^{2}=1.94$ bar. Thus, a vacuum pump of pressure 1.94 bars is minimum requirement.

3.5 Block Diagram of Our Motor - CNC Shield - Arduino Interface

The Connections for stepper motor and CNC shield with the Arduino are given in the below circuit diagram.

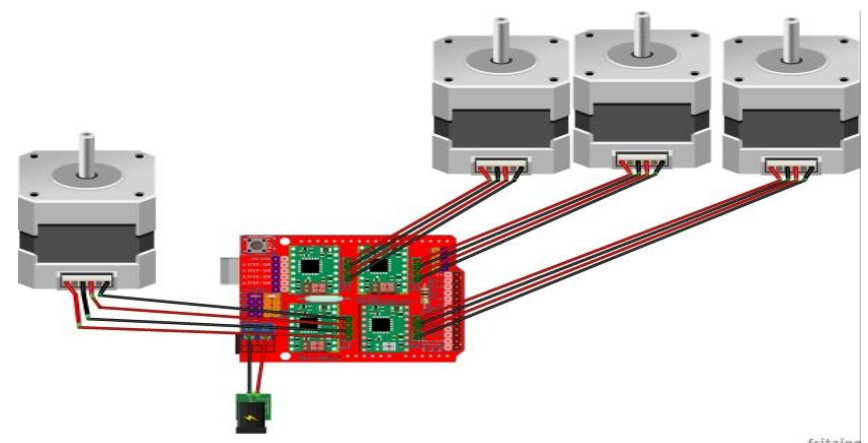

Fig.3.Block Diagram of Our Motor - CNC Shield Arduino Interface
The above circuit diagram shows the connection of 4 stepper motors with the motor drives mounted on the CNC shield. The SPMS is also connected with the pins in the CNC shield. The Block Diagram of the Entire connection and interface is given in the Fig.3. This is explained below.

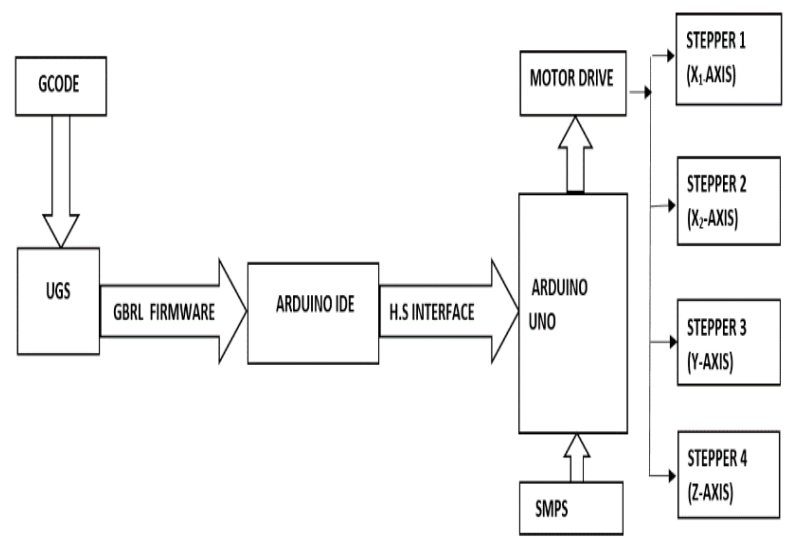

Fig.3.The Block Diagram of the Entire connection and interface

The G-Code that is generated using the coordinates on the PCB is uploaded to the Universal G-Code Sender (UGS) which sends the G-Codes as a GBRL firmware that translates the Codes to corresponding stepper motor pulse in Arduino IDE's format.

\subsection{G-CODES AND ITS COORDINATES}

G-Codes moreover referred to as prerequisite codes, CNC program starts with alphabet G. In general, it is a code that commands the machine tool what kind of operation to be done such as:

- Rapid movement.

- Controlled feed-in arc or straight line.

- A sequence of guided feed movements which may cause a hole to bore, a work piece routed to a specific dimension, or a contour shape added to that edge of job

- Set the tool information, e.g. offset.

- Change system coordinates.

The coordinates are determined by the SMD component position on the PCB. The G-Codes are produced for the Corresponding coordinates.

\subsection{GBRL}

GBRL is a CNC controller and its configuration are given below 
$\$ 0=10$-Step pulse, microseconds

$\$ 1=25-$ Step idle delay, milliseconds

\$22=1-Homing cycle, Boolean

$\$ 23=0$-Homing dir. invert, mask

\$24=25.000-Homing feed, $\mathrm{mm} / \mathrm{min}$

$\$ 100=250.000-X$ steps $/ \mathrm{mm}$

$\$ 101=250.000-Y$ steps $/ \mathrm{mm}$

$\$ 102=250.000-Z$ steps $/ \mathrm{mm}$

\subsection{UGS (Universal G-Code Sender)}

A fully functional G-Code system that is used to connect advanced CNC controllers like GRBL and TinyG. Universal G-Code Sender can be a selfcontained Java program with all external dependencies, meaning the rest will be supported if you have the UGS setup for the Java Runtime atmosphere.

\subsubsection{Features of UGS}

\subsubsection{Toggles and Overrides}

Just version of the platform. Additional Overrides app can easily monitor real-time feed and speed overrides from the window menu.

\subsubsection{Mode Jog}

This excellent jog mode ensures that the GCODE state will not be revised and also allows you to avoid jogging while it is underway. UGS automatically uses this new syntax when it detects a supported version of GRBL. The STOP action is used to stop the process during a jogging usage.

\subsection{Homing and Calibration}

The position to which the machine returns after a particular cycle is called Home position and to set the home position initial coordinates are given. The calibration of the machine is done with the help of a simple ruler. A ruler is set in the path and particular steps per revolution are given to check the accuracy and precision of the stepper motor and calibrated accordingly.

\subsubsection{Hardware and Software (H.S) Interface}

H.S interface is the interaction between the software (Arduino IDE) and the Hardware (ARDUINO) via Arduino cable.

\subsection{Performance}

\subsubsection{Micro Stepping}

Micro stepping is an approach for smoother transmission of the stator flux of a stepper than in single-or half-stage drive modes.

- This means noise measures down to $0 \mathrm{~Hz}$. It also represents a smaller step.

- The angles and the best possible positioning are achieved.

- Several different micro-stepping modes exist, with step lengths from maximum step down to or even less than $1 / 32$ micro-step.

- Synchronous electric motor is a stepper motor. That means the stable stop position of the rotor is in equilibrium with the flux of the stator coil. The rotor is machined to rotate Torque $(\mathrm{TH})$ and the distance between the stator flux (fs) and the rotor position (fr).

- $\mathrm{T}=\mathrm{TH} * \sin (\mathrm{fs}-\mathrm{fr})$ where the electrical degrees of fs and fr are given.

- The stator flux is rotated at 90 and 45 electrical degrees respectively each step of the motor when the stepper is driven in full-step and half-step mode.

From the formula above we can see that the motor develops a pulsing torque.

\subsection{Testing}

When the Trial run was done for placing the SMD components on PCB for soldering, up to 100 components were soldered per hour. The placement rate was improved for successive trial runs. The major problems faced during the testing phase were reduced belt tension, port disconnectivity improper calibration.

\section{Conclusion and Future Scope}

Our project focuses on developing a portable and efficient Pick and place for small scale industries 
www.rspsciencehub.com

with high precision and accuracy by making use of a compact design, reliable controller and an economic setup for vacuum suction.

\section{Future Scope}

Image processing can be implemented for Board placement. It automatically generates the coordinates of a given PCB with the help of a camera module.

It also helps in the accurate placement of SMD components and acts as a poka-yoke system (machine cuts of when PCB is not in position).

A different controller can be used to make the machine more efficient in terms of speed and new features. SMOOTHIEBOARD controller can be used instead of Arduino which has connections for Ethernet and USB and works, two TMC2660 stepper motor drivers with $1 / 256$ micro-stepping at $120 \mathrm{Mhz}$. It includes a ROM of $512 \mathrm{kB}$ and $64 \mathrm{kB}$ RAM.

Provision for the Automatic tool change mechanism at Z-Axis for the usage of multiple tools can be given.

Software to auto-generate G-Codes with the PCB Design interface software and a GUI (Graphical User Interface) can be developed which generates the G-code for the selected PCB.

\section{References}

1) I. Nae and T. Andrei, "Designing and building a CNC router using stepper motors", Serial Technical, vo. LXII, pp. 55-62, 2010.

2) K. Nagai, "Learning while doing: Practical robotics education", IEEE Robotics \& Automation Magazine, vol. 8, pp. 38-43, June 2001.

3) Linggarjati, Jimmy, and Rinda Hedwig. "Manually interchangeable heads of homemade computer numerical control (CNC) machine." internetworking Indonesia journal 1.1 (2013).

4) V.K. Pabolu and K.N.H. Srinivas, "Design and implementation of a three -dimensional CNC
Volume 02 Issue 08 August 2020

machine", Int. J. Computer Science and Engineering, vol. 2, pp. 2567-2570 2010.

5) Surface Mount Technology with Fine Pitch Components (The manufacturing issues) by $\mathrm{H}$. Danielson.

\section{6) http://openpnp.org/hardware/}

7)Total Pressure Measurements in Vacuum Technology. 